\title{
Influence of Pipe-Diameter on Water Hammer
}

\section{Phenomenon}

\author{
Provenzano, Pablo Gabriel \\ Departamento de Ingeniería e Investigaciones Tecnológicas, Universidad Nacional de La Matanza, San Justo (C.P.1744), Provincia \\ de Buenos Aires, Argentina
}

\begin{abstract}
The influence of parameters pertaining to the confinant structure on water hammer had been less studied than those relative to the fluid. One of them is the inner pipe-diameter, a basic structural-parameter that makes its influence in essential hydraulic topics such as head loss, in pipelines. In this paper, the objective is to analyze the inner-diameter influence on water hammer phenomenon. An analytical algorithm for solving the unsteady-one-dimensional water hammer model had been applied. It had allowed estimating the instantaneous head at any point of a single pipeline. The model was solved by mean of the Laplace's Transformed application and the anti-transforming procedure into the complex field. To determinate the influence of internal-diameter conduit on the pressure oscillation, four distinct inside-diameter values were introduced into the solution, successively. The first overpressure-peak at each case was tabulated along with the corresponding inner-diameter and a mathematical relation had been founded. The obtained results show a close dependence between both, over-pressure peaks and internal-pipe diameter. It was founded that this dependence is given in terms of a non-linear relation between them. It was further founded that the wave frequency is sensitive to the variation of the pipe-diameter.
\end{abstract}

Key words: Transient, overpressure, shear stress, radial.

\section{Nomenclature}

$a: \quad$ Wave-speed $(\mathrm{m} / \mathrm{s})$

$D: \quad$ Internal diameter of pipe $(\mathrm{m})$ or $(\mathrm{mm})$

$F$ : $\quad$ Lost charge coefficient $\left(\mathrm{kg} / \mathrm{m}^{3} . \mathrm{s}\right)$

g: $\quad$ Earth gravity celerity $\left(\mathrm{m} / \mathrm{s}^{2}\right)$

$H$ : $\quad$ Transient pressure measured as piezométric head (m)

$H_{0}: \quad 1$ st transient pressure peak (at $\left.t=\tau\right)(\mathrm{m})$

$L: \quad$ Conduit length $(\mathrm{m})$

$P$ : $\quad$ Transient pressure in temporal dimension $(\mathrm{Pa})$

$P_{0}$ : $\quad$ Static pressure $(\mathrm{Pa})$

$s: \quad$ Complex variable at Laplace's Transformed field.

$t$ : Time (s)

$V: \quad$ Instantaneous fluid velocity $(\mathrm{m} / \mathrm{s})$

$V_{0}$ : Stationary fluid regime velocity $(\mathrm{m} / \mathrm{s})$

$x$ : One-dimensional axis (m)

\section{Introduction}

Water Hammer is basically defined as a fast transient that begins when the steady state flow conditions in a

Corresponding author: Provenzano, Pablo Gabriel, Ph.D., researcher, research fields: fluids mechanics, transient fluids, water hammer phenomenon. E-mail: pprovenzano@ing.unlam.edu.ar. confined fluid are perturbed. Pressure oscillation is a typical flow response when those perturbations are triggered. That oscillatory behavior could be described as an alternated succession of pressure-peaks and deeps, and the last ones may reach the vapor-tension-level. The latter is responsible for generation of both negative phenomena: cavitation and column separation. In several cases, pressure-oscillation is cause of mechanical vibrations, noises, sudden movement along conduits and branches, and efficiency decrement in operating systems are frequently reported. In addition, the pressure-deeps above mentioned could reach as low values as to invalidate the homogeneity and continuity flow assumptions.

Those constitute water hammer evidences. Another consequence, such as wall shear stress (in pipes) and cushion and bushing resentment has got less visible.

Bibliography about Water Hammer has covered the last 160 years. Contributions from different scientist and researchers are extensive: from the pure theoretical 
view (for example, analytical attempts via the Heavyside Method) to empirical experiences and numerical ones (like the application of Numerical Methods since the last five decades) [1-3].

On water hammer analysis the efforts were set mainly into the analysis of fluid behaviour, in the last decades. Numerous researchers had attempted to improve the pressure-wave attenuation along the time trying to the introduction of different friction terms into the water hammer model and solving it using the numerical method referred, and others. In the last decades, the production volume on that appointed aspect was resulted higher than the other ones.

In this sense, six different investigation-lines about the inclusion of unsteady friction term into the model had been developed [4, 5].

Other parameters, pertaining to the pipeline, as inner-pipe diameter, have received less attention than the typical ones of fluid. However, fluid velocity (and so, fluid flow) is conditioned (since the conduit crosssection area) by pipe-diameter, as expressed in the mass balance formulation [6], and fluid velocity shows continuous changes during water hammer episode. Therefore, pipe diameter must have a direct incidence respect to this phenomenon.

A concept known as FSI (fluid-structure-interaction) has gained influence progressively on water hammer study, basically in the two last decades. This mentioned concept includes structural parameters like pipe material, wall-thickness, and inner-diameter into the analysis obtaining a slightly-larger celerity values than those calculated from classical celerity formula $[7,8]$.

This work deals with the incidence of internal pipe-diameter on the phenomenon. Pipe diameter is a useful variable in pipeline and structures that conveying fluids and it is easy to measure. Diameter datum is included into all technical device and installation data.

The objective of this work is to describe the dependence of pressure-wave respect to the inner-pipe diameter, and quantify those by mean of a mathematical relation that hurling some light about the magnitude that pressure peaks assumes as function of diameter variation. In order to reach this purpose the one-dimensional water hammer model had been boarded through analytical tools as Laplace's Transformed, and an exact formulation of contour conditions. Since those, an analytical algorithm was developed to arrive at the pressure expression at frequencies field. The anti-transformed way was approached by employing to Cauchy's Theorem conclusions in the complex field and a developing in Laurent's Series form, for arriving to the transient pressure solution at temporal field.

This solution was applied to analyze the pressure response in terms to the conduit-diameter values, introducing different values (at each time) into the algorithm, and solving.

Results obtained show a non-linear relation between first overpressure peak (the most critical) and the inner pipe-diameter.

The possible influence of pipe diameter on pressure-wave cycles-number had been analyzed too, and it was founded an inverse relation between them.

\section{Model Formulation}

The analysis of water hammer was done under the following assumptions:

- One-dimensional flow;

- The pipe is water fulfill and remains at this condition during the transient;

- The behavior of both, the liquid and the pipe-wall is lineally elastic;

- There is neither gas nor vapor bubbles generation nor separation of liquid column;

- Horizontal pipe-slope, with constant diameter.

The following one-dimensional wave-equation set defines the analytic pattern of water-hammer model:

$$
\begin{gathered}
\frac{\partial p}{\partial x}+\delta \frac{\partial V}{\partial t}+F V=0 \\
\frac{1}{a^{2}} \cdot \frac{\partial p}{\partial t}+\delta \frac{\partial V}{\partial x}=0
\end{gathered}
$$




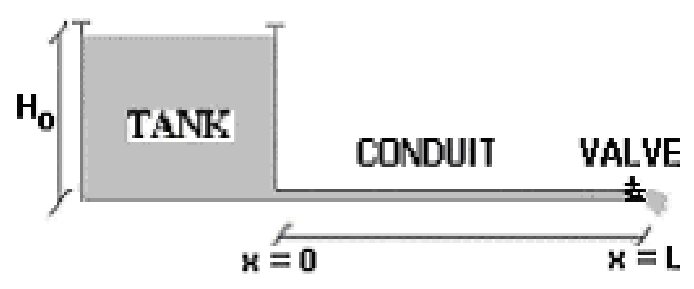

Fig. 1 Analyzed hydraulic-single-system. Horizontal slope and constant inside diameter.

Eq. (1a) (movement) is a differential- forces-balance result. It is obtained from the 2nd Newton Law applying to a fluid element in a control- volume. Eq. (1b) (continuity) is a differential mass balance result, applied to the same fluid element in a control-volume [9].

\section{Resolution of Model}

Resolution of model was arranged by mean of the application of Laplace Transform to the model (Eqs. (1a) and (1b)). The following expression was obtained:

$$
\bar{P}=A \cdot \cosh \left(\frac{s x}{a}\right)+B \cdot \sinh \left(\frac{s x}{a}\right)+\frac{P_{0}}{s}
$$

Eq. (2) is the general expression for pressure at frequencies field.

At this generic expression, $P$ represents the

$$
\begin{aligned}
& p(x, t)=P_{0}-V_{0} F x+ \\
& +\frac{\pi \cdot V_{0}}{Q \cdot L} \cdot \sum_{n=1}^{\infty}(-1)^{n-1} \frac{\left[\left(\psi^{2}+\vartheta^{2}\right)^{\frac{1}{2}}+\vartheta\right] \sin \left[\left(\psi^{2}+\vartheta^{2}\right)^{\frac{1}{2}} \cdot t\right] \sin \left[(2 n-1) \frac{\pi x}{2 L}\right] \cdot e^{-\frac{\vartheta}{2} \cdot t}}{\sqrt{2}\left\{\left[\psi^{2}+\left(\frac{\vartheta}{2}\right)^{2}\right]^{\frac{1}{2}}+\left(\frac{1}{2 \sqrt{2}}-\frac{1}{2}\right) \vartheta\right\}^{2}-\left(\frac{1}{2 \sqrt{2}} \cdot \vartheta\right)^{2}}
\end{aligned}
$$

where, $\psi=\left[(2 n-1) \frac{\pi a}{2 L}\right] ; \quad \vartheta=\frac{F}{\delta}$

Eq. (4) is the solution for pressure $(p(x, t))$ at temporal field.

\section{Water Hammer Simulation}

Fig. 2 shows an empirical transient-pressure curve (black continuous line [10] and the result of analytic model by mean of the application of solution (Eq. (4) transient-pressure in Laplace's Transform field. The particular solution to the analyzed system is reached introducing the boundary conditions into the developing algorithm, to solve the $A$ and $B$ integration constants. Boundary conditions are:

for $t>\tau, t=0, V=V_{0}$;

for $t \geq 0$, at $x=0, P=P_{\text {static }}=$ constant;

for $0<t \leq \tau$, at $x=L, V=V(t)$ (closing law).

Since those, and applying the same procedure, it arrives to the next formula:

$$
\bar{P} ? \frac{\rho \cdot a}{g} \cdot \frac{V_{0} \cdot \sinh \left(\frac{s x}{a}\right)}{s \cdot \cosh \left(\frac{s L}{a}\right)}+\frac{P_{0}}{s}
$$

Eq. (3) is the particular expression for pressure at frequencies field.

\subsection{Anti-transformation}

It is searching for the solution that describes pressure behavior under the transient phenomenon at temporal field. The anti-trasformation procedure is an analytical algorithm that operated into the complex field allows obtaining the sought solution. Applying those to Eq. (3), it arrives to the next equation: 
It observes a close modeled in wave-oscillation amplitude and no-phase displacement, displaying a good agreement between both, the empirical and analytical curves.

\section{Internal Diameter Influence on Water Hammer Magnitude}

Different variables affect water hammer expression, in a direct or indirect way. For example, inside conduitdiameter is involved into the transient since it takes a direct influence on friction characteristics (that it is an important wave-attenuator factor) as well as an indirect interaction form the wave speed (inner diameter makes a decisive influence on wave speed magnitude, and this makes a clear interference on transient pressure amplitude).

First, it had analyzed the influence of pipe-diameter onto the pressure wave amplitude. In order to estimate the relationship between them and to quantify those, transient-pressure oscillations had been modeled for four different internal pipe-diameters. The first one corresponding to the $94 \mathrm{~mm}$ empirical-pipe-diameter size, pertains to the analyzed system [10]. The following three are: this empirical diameter-size and a half, two empirical diameter-size, and a half diameter-size respect to the empirical one.

Those values were used to calculate the corresponding wave speed values, as shown in Table 1 .

Next, each wave-speed values was applied to model the transient-pressure at each tested case.



Fig. 2 Pressure peaks and deep as function of time in water hammer episode.
Table 1 Inner pipe-diameter and its respective wave speed to the analyzed system.

\begin{tabular}{lll}
\hline Case & Inner pipe diameter $(\mathrm{mm})$ & Wave-speed $(\mathrm{m} / \mathrm{s})$ \\
\hline 1st & $94.00($ empirical value $)$ & 270.00 \\
2nd & 141.00 & 223.29 \\
3rd & 188.00 & 193.95 \\
4th & 47.00 & 378.00 \\
\hline
\end{tabular}

\section{Results and Discussion}

Fig. 3 shows water hammer modeling for the three latest cases that are detailed in Table 1.

In order to maintain graphical clearness, the first case modeled (Table 1) had not been included into this figure. Instead, that case has previously showed together with the empirical curve in Fig. 2.

It could be distinguished three-modeled curves (colored) in Fig. 3. Each curve displays the pressure oscillations during water hammer in the analyzed system, for each internal-diameter studied. Two hard observations arise in Fig. 3: the first is referring to a clear changing in pressure-wave amplitude, as function of the pipe-diameter submitted values (Table 1). For example, it could see that the lower wave-amplitude (red curve) is corresponding to the larger pipe-diameter.

In the way to establishing the kind of relation that links both variables, the even values the first pressure peak (the most critical) and each corresponding inner-pipe diameter had been plotted in Fig. 4.

The study about the relation that links these variables shows that a non-linear dependence had been founded. This one consists in a decreasing exponential approaching and it is expressed by the included formula at the upper right margin, in the cited figure. This exponential relation had been tested by means of the determination coefficient value $\left(R^{2}\right)$.

The calculated value to the mentioned coefficient is $R^{2}=0.991$ (close to 1 ). This is aiming to an exponential trend for pressure peaks when pipe-diameter assumes increasing values.

The other observation is the changes in pressure-wave frequency as function of each pipe-diameter size. 


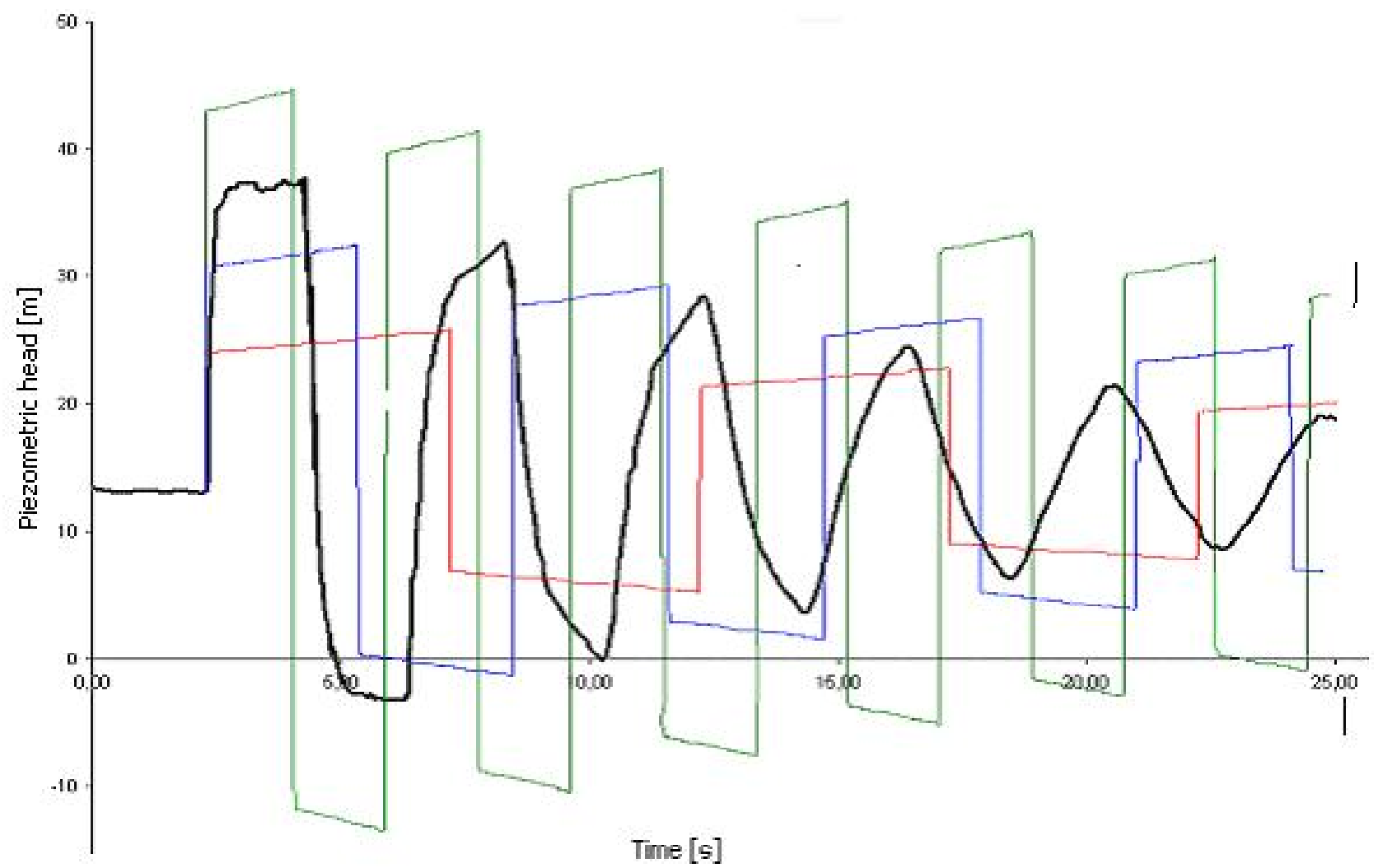

Fig. 3 Empirical water hammer-curve (black line) and water hammer modeling for three different wave speed values: 193.95 $\mathrm{m} / \mathrm{s}$ (red line), $223.29 \mathrm{~m} / \mathrm{s}$ (blue line) and $378 \mathrm{~m} / \mathrm{s}$ (green line).

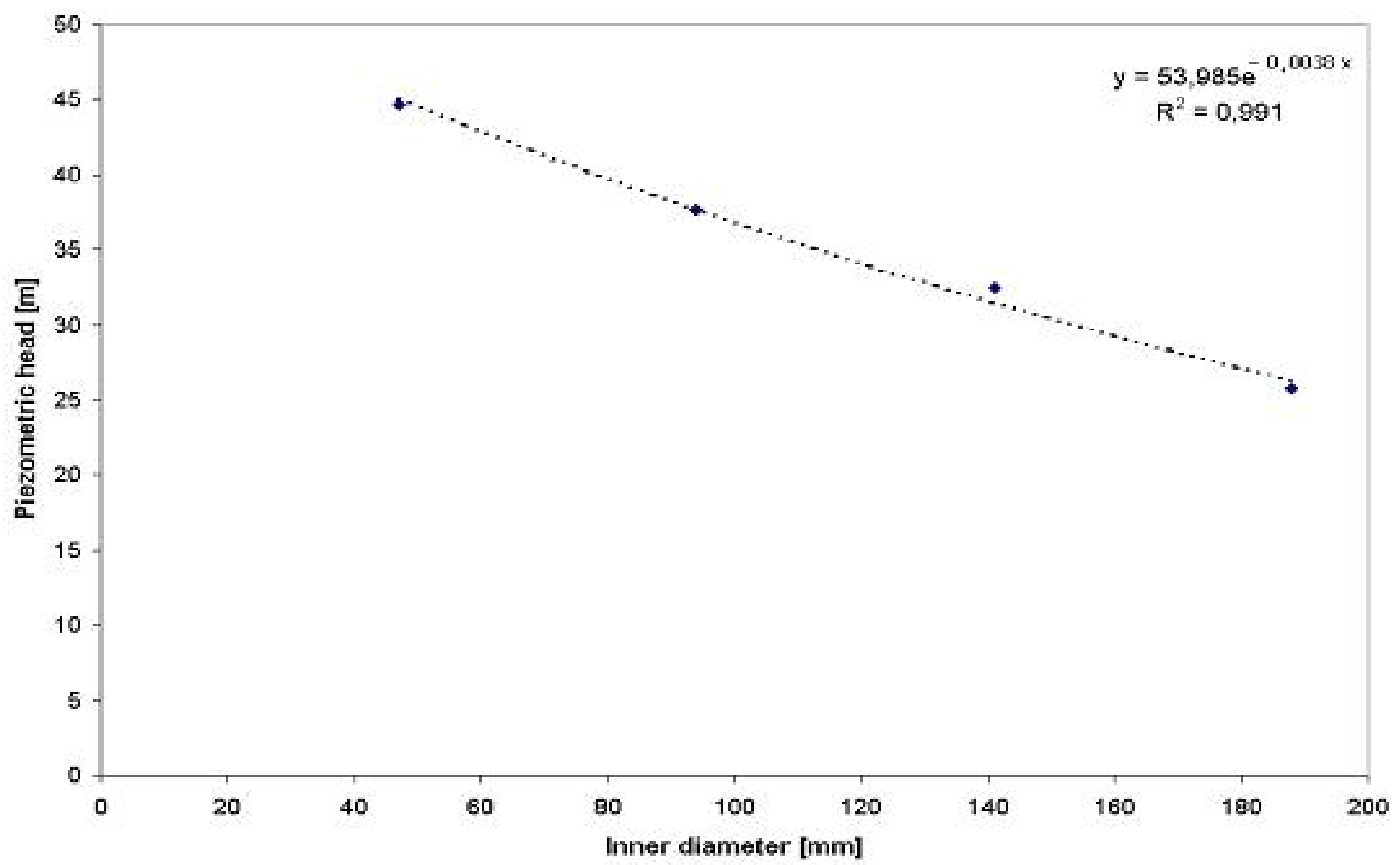

Fig. 4 First pressure peak $(\mathrm{m})$ as function to inner pipe diameter ( $\mathrm{mm}$ ) (blue points) and decreasing exponential curve trend (dotted line). 
Referring to this when a minor value is assigned to it, an increment in cycles (measured at the same time-interval) takes place.

For example, empirical water hammer graphic (black line) from Fig. 3 (where diameter size is $94 \mathrm{~mm}$ ) shows five complete cycles in the $25 \mathrm{~s}$ time-interval, while the water hammer tested (at the same system) for a $47 \mathrm{~mm}$ pipe-diameter size (a half diameter size respect to the previous case), that is represented by the green curve, accounts six complete cycles, in an equal time-interval. In the same way, two complete cycles and a quarter for a $188 \mathrm{~mm}$ pipe-diameter (red curve) and three and a half cycles (blue line) for $144 \mathrm{~mm}$ ones have acknowledged in it (Fig. 3).

Pressure-wave frequency is a parameter whose major application treatment had founded in phenomenon-attenuation analysis, such as the friction effect as frequency dependent [11, 12]. However, pressure-wave frequency has an important incidence from another side since each pressure-wave cycle represents a strike against the valve and the neighborhood structures.

The confined water into the pipe, like a true compact block, is oscillating giving several impacts against the valve and any components, until the phenomenon had got vanish, and therefore, as more strikes the valve has endured (at an equal time-interval) as more risk to damage is on this one. Practically the cycles-account, from this perspective, is not reported in water hammer published literature, however it's a useful parameter to examine the confinant-structure performance undergone to oscillating pressure due to the direct relation between strikes-quantity and stress-increment and to easily-to-measure of that.

\section{Conclusions}

Those results reached above are indicating the sensitiveness of water hammer magnitude respect to the conduit-diameter variation. Conduit-diameter has been affecting the transient-pressure amplitude. The decreasing exponential trend founded (Fig. 4) is pointing out an incremented ratio in pressure-wave amplitude in relation to the decrement in conduit-diameter and it should not be unawares since the pressure-amplitude increment means more vulnerability affecting the fluid- structure.

It is founded too that the frequency wave is affected when pipe-diameter is shifted. This parameter is an indicative to water-block strikes (cycles account) against the valve in a fixed interval-time. If conduit-diameter is diminished, the strikes are incremented for a same service (Fig. 3) and it is hard because the induced stress-on-valve (and so, the lasting-life of valve) is not only strongly dependent on the pressure-wave amplitude, but the strikes quantity in each transient event, too.

Those are meaning that the cycles-account is a significant parameter that should be included into the phenomenon analysis.

In view to the present analysis and previous works, the pipe and accessories selection should be not rest ringed to economic matters as a unique aspect to consider, rather making the material choices from those physical conceptions too. Bigger internal conduit-diameter (and accessories) and lesser rigid conduits materials as possible to employ are good criteria to apply at the moment to make a system design or develop maintenance-task.

\section{References}

[1] Hager, W. 2001. "Contribution Suisse á la Théorie du Coup de Belier." Journal of Hydraulic Research 39: 3-10.

[2] Murga, N., and Molina, N. 1997. "Sistema de Protección de Bombas y Cañerías en Oleoductos Sometidos a Flujos Transitorios." Universidad Nacional del Sur, Bahía Blanca.

[3] Tijsseling, A., and Anderson, A. 2007. "Johannes von Kries and the History of Water Hammer." Journal of Hydraulic Engineering 133 (1): 1-8.

[4] Bergant, A., and Simpson, A. 2001. "Developments in Unsteady Pipe Flow Friction Modelling." Journal of Hydraulic Research 39 (3): 249-57.

[5] Vardy, A., and Hwang, K. 1991. "A Characteristics Model of Transient Friction in Pipes." Journal of 
Hydraulic Research 29 (5): 669-84.

[6] Abreu, J., Guarga, R., and Izquierdo, J. 1995. "Transitorios y Oscilaciones en Sistemas de Impulsión." Caps. 1; 2; 7 - Unidad Docente Mecánica de Fluidos, Dpto. Ingeniería. Hidráulica y Medio Ambiente, Universidad Politécnica de Valencia-España.

[7] Bergant, A., Tijjseling A., Vitkovsky, J., and Covas, D. 2008. "Parameters Affecting Water-Hammer Wave Attenuation, Shape and Timing." Journal of Hydraulic Research 46 (3): 373-81.

[8] Rocha, G., Bastos, F., and Freitas, R. 2012. "Numerical Solution of Fluid-Structure Interaction in Piping Systems by Glimm's Method." Journal of Fluids and Structures 28:
392-415.

[9] Streeter, V., and Wylie, E. 1988. Mecánica de Fluidos. USA: Mc.Graw-Hill.

[10] Brunone, B. 2000. "Velocity Profiles and Unsteady Pipe Friction in Transient Flow." Journal of Resources Planning and Management 126 (4): 236-44.

[11] Zielke, W. 1967. "Frequency Dependent Friction in Transient Pipe Flow." Journal of Basic Engineering Transaction of the ASME 90 (1): 109-15.

[12] Achard, J., and Lespinard, G. 1981. "Structures of the Transient Wall-Friction Law in One-Dimensional Models of Laminar Pipes Flow.” Journal of Fluids Mechanics 113: 283-98. 